\title{
1 The molecular mechanism of load adaptation by branched actin networks
}

3

4 Tai-De Li $i^{1,2,3^{*}}$, Peter Bieling ${ }^{2,4,5^{*} \dagger}$, Julian Weichsel $^{6}$, R. Dyche Mullins ${ }^{4, \dagger}$ and Daniel A. Fletcher ${ }^{1,2,7 \dagger}$

$6 \quad{ }^{1}$ Department of Bioengineering \& Biophysics Program, University of California, Berkeley, CA 94720, USA

$7 \quad{ }^{2}$ Division of Biological Systems \& Engineering, Lawrence Berkeley National Laboratory, Berkeley, CA 94720, 8 USA

$9{ }^{3}$ Advanced Science Research Center, City University of New York, New York, NY, USA

$10{ }^{4}$ Department of Cellular and Molecular Pharmacology and Howard Hughes Medical Institute, University of 11 California, San Francisco, CA 94158, USA

$12{ }^{5}$ Department of Systemic Cell Biology, Max Planck Institute of Molecular Physiology, Dortmund, Germany

$13{ }^{6}$ Department of Chemistry, University of California, Berkeley, 94720, USA

$14 \quad{ }^{7}$ Chan Zuckerberg Biohub, San Francisco, CA 94158, USA

15

16

$17 *$ equal first authors

$18 \nmid$ co-corresponding authors (dyche.mullins@ucsf.edu, peter,bieling@mpi-dortmund.mpg.de, fletch@berkeley.edu)

19 


\section{Abstract:}

22 Branched actin networks are self-assembling molecular motors that move biological membranes

23 and drive many important cellular processes. Load forces slow the growth and increase the

24 density of these networks, but the molecular mechanisms governing this force response are not

25 well understood. Here we use single-molecule imaging and AFM cantilever deflection to

26 measure how applied forces affect each step in branched actin network assembly. Unexpectedly,

27 force slows the rate of filament nucleation by promoting the interaction of nucleation promoting

28 factors with actin filament ends, limiting branch formation. This inhibition is countered by an

29 even larger force-induced drop in the rate of filament capping, resulting in a shift in the balance

30 between nucleation and capping that increases network density. Remarkably, the force

31 dependence of capping is identical to that of filament elongation because they require the same

32 size gap to appear between the filament and load for insertion. These results provide direct

33 evidence that Brownian Ratchets generate force and govern the load adaptation of branched actin

34 networks. 


\section{Introduction}

37 Unlike conventional motor proteins, which produce force by converting chemical energy into conformational changes, branched actin networks generate force by spatially and temporally coordinating interactions between a set of four core components: (i) a nucleation promoting

40 factor (NPF) related to the Wiskott-Aldrich Syndrome Protein (WASP), (ii) the Arp2/3 complex,

41 (iii) capping protein (CP), and (iv) profilin-actin complexes (Achard et al., 2010; Akin and

42 Mullins, 2008; Bieling et al., 2018; Loisel et al., 1999). Branched actin network assembly begins

43 when signaling molecules, such as Rho-family GTPases, cluster together and activate NPFs on a

44 membrane surface (Dominguez, 2009; Husson et al., 2010). Active NPFs locally promote actin

45 nucleation and branching by the Arp2/3 complex (Mullins et al., 1998; Rohatgi et al., 1999).

46 These newly created actin filaments elongate at their fast-growing (barbed) ends from profilin-

47 actin complexes (Funk et al., 2019), fed by an intrinsic polymerase activity of the nucleation

48 promoting factors (Bieling et al., 2018). Individual filaments elongate and push against the NPF-

49 coated membrane surface, but only for a short time before capping protein terminates their

50 growth (Edwards et al., 2014; Schafer et al., 1996). As a result, steady-state network growth

51 requires continual nucleation. Whenever active NPF molecules are concentrated together on a

52 membrane surface, this sequence of interactions is sufficient to create a powerful molecular

53 motor capable of generating kilopascal $\left(\mathrm{nN} / \mu \mathrm{m}^{2}\right)$ pressures (Bieling et al., 2016; Marcy et al.,

54 2004; Parekh et al., 2005).

The response of a molecular motor to force is fundamental to understanding its biophysical mechanism. Force, for example, coordinates the out-of-phase stepping of two-headed kinesins along a microtubule (Yildiz et al., 2008), and it can cause dynein motors to step 'backward,' toward the microtubule plus end (Gennerich et al., 2007). Force also causes some myosin motors to cling more tightly to actin filaments (Laakso et al., 2008). Similarly, force produces dramatic effects on the motor activity of branched actin networks, increasing the number and density of actin filaments (Bieling et al., 2016; Mueller et al., 2017) as well as the mechanical efficiency of the network (Bieling et al., 2016). These changes in filament density also optimize the material

64 properties of a branched actin network to better resist deformation and to generate higher forces.

65 Load adaptation in a growing actin network comprises two distinct processes: (i) reorientation of 
67 increase in the steady-state number of growing filaments (Bieling et al., 2016). Filament

68 reorientation has been explained by kinetic competition models (Weichsel and Schwarz, 2010)

69 but the mechanism underlying the force-induced increase in filament number remains unknown.

71 To figure out how load forces increase the number of growing filaments, we studied branched

72 actin networks assembled from purified proteins on micro-patterned, functionalized glass

73 surfaces. We measured the rate of network growth against defined forces using a modified

74 Atomic Force Microscope (AFM) and simultaneously visualized the flux of constituent

75 molecules -actin, capping protein, and the Arp2/3 complex- into the network by total internal

76 reflection fluorescence (TIRF) microscopy (Bieling et al., 2016). From these measurements, we

77 find that load adaptation in the network arises from a mismatch in the force-dependent activities

78 of capping protein and the Arp2/3 complex. Contrary to our expectations, new branch formation

79 is reduced by load-dependent interaction with nucleation promoting factors on the membrane, a

80 process we refer to as 'barbed end interference'. However, rates of capping are reduced even

81 more significantly, leading to a net increase in actin density with increasing force. Interestingly,

82 we show that the force responses of filament elongation and capping are closely matched,

83 ensuring that the average filament length and molecular stoichiometry of the network remain

84 constant across a wide range of load forces. The exponential force response of filament capping

85 suggests that it is governed by a Brownian Ratchet similar to the one that is thought to govern

86 filament elongation. To test this idea directly we created a 'bulky' capping protein mutant that

87 adds a larger length increment to the barbed end of an actin filament. When added to freely

88 growing actin filaments in solution, this bulky mutant shows no defects in capping activity, but

89 when added to actin networks growing against a load force it exhibits a dramatically different

90 force response and cannot keep pace with wildtype capping protein. Overall, our work reveals

91 that a combination of matched and mismatched force responses work together to stabilize

92 branched actin networks and enable them to respond to changing load forces.

94 Results:

95 Load forces produce different effects on actin filament nucleation and capping.

96 The steady-state density of free barbed ends $\left(\mu \mathrm{m}^{-2}\right)$ at the growing surface of a branched actin

97 network is determined by the ratio of the nucleation rate across that surface $\left(\mu \mathrm{m}^{-2} \mathrm{sec}^{-1}\right)$ divided 
by the per-filament capping rate ( $\mathrm{sec}^{-1}$; (Mullins et al., 2018)). To change the density of growing filament ends, load forces must somehow alter the balance between nucleation and capping.

100 Some numerical simulations propose that the rate of filament nucleation increases under load as

101 a result of the autocatalytic nature of Arp2/3 branching (Carlsson, 2003), but this has never been

102 tested experimentally. Furthermore, as far as we can tell, the force-dependence of filament

103 capping has never been investigated or incorporated into numerical simulations.

We measured the rates of filament nucleation, elongation, and capping in branched networks under load by performing TIRF microscopy on fluorescently labeled Arp2/3 complexes, actin, and capping protein (Figure 1A, B) as these molecules incorporated into networks growing from coverslips printed with square patterns of the C-terminal, Arp2/3-activating region of the nucleation promoting factor WAVE1 (WAVE1 $\Delta N)$. We applied defined load forces using a calibrated AFM cantilever and found that the density of all three network components increased

111 monotonically with increasing loads (Figure 1C). We calculated the rate of Arp2/3 complex

112 incorporation by multiplying the average fluorescence intensity in the TIRF field (Figure 1B) by

113 the steady-state network growth velocity under each loading condition (Figure 1C). Contrary to

114 previous predictions, the rate of Arp2/3 incorporation decreased linearly with applied force

115 (Figure 1D), falling to approximately $50 \%$ of its initial value at high load ( 1200 Pa).

117 To quantify the effect of force on filament elongation and capping, we also multiplied the 118 average fluorescence intensities of labeled actin and capping protein by the network growth rate.

119 Because both actin and capping protein interact with free filament ends whose number increases

120 with load force, we also normalized the bulk incorporation rates by the free barbed ends densities

121 produced under the same loads (Figure 1C; (Bieling et al., 2016)). This analysis revealed a

122 dramatic, force-induced reduction in the rates of filament elongation and capping (Figure1 D),

123 which can be fit by a double exponential decay. When load force is converted to force per

124 filament, however, the responses of both elongation and capping are well fit by a single

125 exponential decay (Supplemental Figure 1), suggesting that the processes are governed by the

126 same mechanism. Importantly, the drop in filament capping outstrips the drop in nucleation rate, 127 causing the nucleation/capping ratio to increase with increasing force (Figure 1E). We conclude, 128 therefore, that the force-induced increase in filament number is driven by the mismatched force 
responses of filament nucleation and capping rates, not by a force-dependent increase in the rate

130 of nucleation.

132 Our measurements are not explained by a previous model for the effect of force on actin

133 nucleation (Carlsson, 2003), so we investigated the molecular mechanism responsible for this

134 effect. We first visualized incorporation of individual Arp2/3 complexes into growing networks

135 (Figure 2A) using TIRF microscopy. For these single-molecule experiments, we mixed trace

136 amounts of fluorescent Arp2/3 with a large excess of unlabeled complexes (1:5000) and used this

137 mixture to form branched actin networks from WAVE1 $\Delta \mathrm{N}$-coated surfaces. At this low labeling

138 ratio, individual fluorescent Arp2/3 complexes appeared abruptly as diffraction-limited spots on

139 the WAVE1 $\triangle \mathrm{N}$-coated surface and then faded as they moved with the growing actin network

140 away from the coverslip surface and out of the TIRF illumination field (Figure 2A, Supplemental

141 Movie 1). We classified spots whose intensity decayed exponentially with time as network

142 incorporation events, and we rejected fluorescent spots that disappeared in a single step due

143 either to dissociation or photobleaching. Based on the measured surface density of NPFs in our

144 assay (1850 um $\mathrm{um}^{-2}$, (Bieling et al., 2018)), we determined a nucleation rate of $0.037 \mathrm{~s}^{-1}$ per

145 WAVE1 $\triangle \mathrm{N}$ molecule. This rate is notably faster than the limiting step in Arp2/3 branching

146 observed in solution assays (Helgeson and Nolen, 2013; Smith et al., 2013; Zalevsky et al.,

147 2001), demonstrating that nucleation within a branched network is surprisingly rapid. However,

148 because release of the NPF from the nascent branch appears to limit nucleation in solution

149 (Helgeson and Nolen, 2013; Smith et al., 2013), we speculate that retrograde forces generated by

150 network growth facilitate dissociation of the surface-bound NPF and accelerate nucleation in the

151 context of a force-generating network.

153 Increasing load forces caused the fluorescence decay of individual Arp $2 / 3$ molecules to slow

154 down, as expected from load-dependent slowing of network movement (Figure 2A,

155 Supplemental Movie 1, (Bieling et al., 2016)). We calculated a time constant for each Arp2/3

156 incorporation event by fitting its fluorescence decay profile with a single exponential (Figure

157 2C). The calculated time constants for each loading condition followed a Gaussian distribution

158 (Figure 2D) whose mean was inversely correlated with network growth velocity (Figure 2E). In

159 these single-molecule measurements, the rate of Arp2/3 complex incorporation decreased 
moderately $(\sim 17 \%)$ with load (Figure $2 \mathrm{~B})$.

Although both single-molecule and bulk fluorescence measurements revealed a decrease in the rate of Arp2/3 incorporation under load (Figure 1D, 2B), the decrease determined by bulk

164 fluorescence $(\sim 50 \%)$ was more pronounced than for single molecules $(\sim 17 \%)$ over the same stress range (0 to $1000 \mathrm{~Pa})$. To see whether this difference reflects rapid dissociation of a fraction of Arp2/3 complexes from the network, we measured the dwell time between appearance and disappearance of each molecule (Figure 2F, Supplemental Methods). Under low and intermediate loads (25 and $255 \mathrm{~Pa}$ ), the distribution of Arp2/3 dwell times is approximately Gaussian, and the mean lifetime increases with decreasing network velocity (Figure 2F). At high loads $(1020 \mathrm{~Pa})$, however, the distribution develops a 'shoulder' of dwell times shorter than

171 expected (Figure 2F). Fluorescence decay curves of these short-lived events resemble truncated

172 exponentials, consistent with abrupt dissociation of the Arp2/3 complex (Figure 2G,H). These

173 events were too frequent to be accounted for by experimental artifacts such as photobleaching

174 and tracking glitches (Figure 2I, Supplemental Figure 2). Taken together, these results reveal that 175 a significant fraction of Arp2/3 complexes dissociate from the network under high load. Whether

176 forces increase the rate of Arp2/3 dissociation as indicated by recent experiments pulling on

177 individual branches (Pandit et al., 2020) or whether the slower rate of network growth under

178 stress simply keeps the ephemeral branches within the evanescent field for a longer time remains 179 to be determined.

\section{Effect of load on actin-WH2 interactions.}

182 Why does compressive loading reduce the rate of Arp2/3-dependent nucleation? In addition to

183 promoting nucleation by delivering actin monomers to the Arp2/3 complex, WASP-family

184 proteins associate with actin filament barbed ends via their $\mathrm{WH} 2$ domain and thus tether

185 dendritic networks to the boundary they push against (Co et al., 2007; Funk et al., 2021). This

186 means that free barbed ends and actin monomers are in direct competition to occupy available

187 NPF WH2 domains (Figure 3A).

189 Because the concentration of surface-proximal free barbed ends rises in a load-dependent manner

190 (Figure 1C, (Bieling et al., 2016)), we asked whether this might reduce the availability of the 
191 NPF for monomer binding. We adapted a recently developed Förster resonance energy transfer

192 (FRET) assay (Bieling et al., 2018) to directly measure partitioning of WH2 domains between

193 actin monomers and filament ends (Figure 3A). Briefly, we labeled a WH2-adjacent site of

194 WAVE1 $\triangle \mathrm{N}$ with a fluorescent donor (Alexa488) and conjugated a non-fluorescent acceptor

195 (Atto540Q) to monomeric actin using a labeling protocol that does not perturb binding to profilin

196 and WH2 domains (Bieling et al., 2018; Funk et al., 2021).

To measure partitioning of WH2 domains between actin monomers and filament ends we micro-

199 patterned glass coverslips with WAVE1 $\triangle \mathrm{N}$, doped with $30 \%$ donor-labeled molecules. We then

200 assembled dendritic networks under zero or high load (1276 Pa) in the absence of quencher-

201 labeled actin (Figure 3B). We simultaneously arrested network growth and inhibited filament

202 capping by adding Latrunculin B and Phalloidin together with myotrophin/V-1, a combination of

203 inhibitors that arrests network growth but preserves free barbed ends (Bieling et al., 2016). At the

204 same time, we also added quencher-labeled actin monomers, bound to Latrunculin B to prevent

205 their incorporation into free filament ends. The quencher-labeled monomers induced a rapid drop

206 in donor fluorescence (Figure 3B, Supplemental Movie 2) as they bound available WH2

207 domains. We interpret donor fluorescence remaining at long time scales to reflect WH2 domains

208 protected from quencher-labeled monomers by interaction with filament barbed ends.

210 To quantify the fraction of WH2 domains bound to free barbed ends, we compared the remaining

211 donor fluorescence of WAVE1 $\Delta \mathrm{N}$ micro-patterns in either the absence or presence of dendritic

212 actin networks (Figure 3C). In unloaded networks, approximately 7\% of WH2 domains are

213 protected from quenching, while under high load the protected fraction increases by $\sim 3.7$-fold, to

$21427 \%$ (Figure 3D). These numbers are in striking agreement with the 3.3-fold increase in free

215 barbed end density of networks under load (Figure 1C, (Bieling et al., 2016)). The 20\% decrease

216 in available $\mathrm{WH} 2$ domains also agrees well with the load-induced 17\% drop in nucleation rate

217 (Figure 2B). In summary, these results verify that applied forces raise the number of free barbed

218 ends that in turn engage an increasing number of nucleation promoting factors in non-nucleating

219 complexes (Funk et al., 2021; Mullins et al., 2018). This type of negative feedback mechanism,

220 which we call 'barbed end interference' (Funk et al., 2021), quantitatively explains the force-

221 induced decrease in Arp2/3-dependent nucleation. 


\section{Filament capping follows the same force response curve as elongation.}

224 When comparing the effects of force on filament elongation and capping (Figure 1D), we noticed

225 an almost identical force dependence, which explains our previous observation that the average

226 filament length in a branched actin network does not change with applied load (Bieling et al.,

227 2016). We confirmed that the average filament length in a growing network decreased as

228 expected with increasing capping protein concentrations (Supplementary Figure 2). Elevated

229 capping protein levels resulted in sparser networks of shorter filaments that grew faster at

230 comparable loads (Supplementary Figure 2). In line with our previous work (Akin and Mullins,

231 2008), we observed that the increase in capping was entirely compensated for by elevated

232 nucleation rates, confirming that capping protein stimulates nucleation in branched networks

233 (Supplementary Figure 2, (Akin and Mullins, 2008; Funk et al., 2021)). Remarkably however,

234 filament length remained force insensitive at all capping protein concentrations tested (Figure

$2354 \mathrm{~A})$.

237 Although not explicitly predicted, a link between filament capping and elongation is implicit in

238 Elastic Brownian Ratchet theories of force generation (Mogilner and Oster, 1996; Peskin et al.,

239 1993). According to these theories, the rate at which a protein binds the end of a filament

240 growing against a boundary depends on how often thermal motion opens a large enough gap to

241 accommodate the incoming molecule (Figure 4B). Intriguingly, based on their structures (Funk

242 et al., 2021; Kim et al., 2010; Narita et al., 2006), it appears that an actin monomer and a capping

243 protein heterodimer (Figure 4D, top and middle) both require nearly the same gap size to bind

244 the barbed end of an actin filament. Altering the size of capping protein should, therefore,

245 change the force response of filament capping.

247 To test this prediction we constructed a 'bulky' capping protein by fusing dimeric glutathione S-

248 transferase (GST) to the C-termini of both $\alpha$ and $\beta$ subunits of capping protein (Figure 4C,

249 bottom). From existing structures, we predict that the gap required for this bulky variant to bind

250 to the filament end should be significantly larger than for wildtype capping protein (Funk et al.,

251 2021; Kim et al., 2010; Narita et al., 2006). Under load, these larger gaps open much less

252 frequently, and so the rate of capping by the bulky variant should decrease much more strongly 
with applied force. We confirmed that the bulky capping protein caps filament barbed ends with wildtype kinetics in solution (Supplemental Figure 3). To directly compare the force sensitivities of wildtype and bulky capping protein under the same conditions, we constructed dendritic actin networks using mostly (90\%) unlabeled wildtype capping protein, doped with small amounts (5\% each) of wildtype and bulky capping proteins labeled with different fluorescent dyes. We labeled wildtype capping protein with tetramethyl-Rhodamine (TMR) and the bulky variant with Alexa-647. Under low load forces both wildtype and bulky capping proteins incorporated into the network, but only wildtype capping protein maintained a constant stoichiometry with actin under high load (Figure 4D). The bulky capping protein mutant was effectively excluded from the network by applied force (Figure 4D-F, Supplemental Movie 3). We tested for tag-specific effects by using a bulky NusA-tag instead of GST-GST at the N-terminus of capping protein, which resulted in a similar force-dependent reduction of incorporation (Supplemental Figure 3). To test whether these results can quantitatively be described by a Brownian Ratchet mechanism, we developed an analytic model of actin network growth under load (see Appendix). Briefly, we assumed that the capping rate declines exponentially with opposing force according to the Brownian Ratchet model with $\mathrm{k}_{\text {cap }} \sim e^{\frac{-f \cdot \delta \cdot \sin \theta}{K_{B} T}}$, where the $K_{B}$ is the Boltzmann constant, $T$ is the temperature, $\theta$ is the contact angle of filaments to the encounter surface, $f$ is the force of one individual filament against the surface, and $\delta$ is the gap size required for incorporation of capping protein. The latter parameter differs for wildtype capping protein and its bulky variant,

272 leading to their differential response to load. The average filament contact angle changes in 273 response to force (Bieling et al., 2016; Mueller et al., 2017; Weichsel and Schwarz, 2010) and

274 was estimated from the actin density and number of free barbed ends at various forces as 275 measured previously (Bieling et al., 2016). To obtain the force $f$ that opposes a single

276 polymerizing filament, we divided the total external force on the whole network by the measured 277 force-dependent number of free barbed ends sharing this load (Figure 1C; Supplemental Figure 278 1; (Bieling et al., 2016)). Finally, we took into account the internal tethering force that arises

279 from the interaction between free barbed ends and NPF proteins on the surface, which in addition 280 to the external load resists network movement (Figure 3). Except for this characteristic tethering 281 force, all parameters are derived from experimental data. Remarkably, even with all but this 282 single free parameter constrained, this model accurately matches the force- dependent change 283 in the relative incorporation of wildtype capping proteins over its bulky variant (Figure 4E). 
284 Furthermore, the characteristic tethering force yielded from this model $(0.3 \mathrm{pN})$ falls within the

285 typical range for weak protein-protein interactions. This data directly demonstrates that the

286 addition of capping protein to the free barbed end of actin filaments in a branched network is

287 size-dependent insertional process, whose force-dependent kinetics are described by Elastic

288 Brownian Ratchet models (Mogilner and Oster, 2003, 1996)

289 Discussion:

290 How free barbed ends interfere with activation of the Arp2/3 complex.

291 One of the most unexpected results of our study is that force actually decreases Arp2/3 complex

292 activity, suggesting that mother filament availability is not a limiting resource for the nucleation

293 reaction. We show that the increased number of free barbed ends generated under load inhibits

294 Arp2/3 complex activity by tying up WH2 domains and decreasing the ability of the NPFs to

295 activate the Arp2/3 complex. Unlike the previously described 'monomer gating' mechanism

296 (Akin and Mullins, 2008), in which free barbed ends compete with NPFs for monomeric actin,

297 'barbed end interference' is based on a direct interaction between NPFs and barbed ends of actin

298 filaments (Funk et al., 2021; Mullins et al., 2018). This sort of negative feedback, in which the

299 products of the nucleation reaction directly inhibit branching, also explains how Arp2/3-

300 dependent nucleation achieves a constant, homeostatic rate despite its autocatalytic nature

301 (Mullins et al., 2018).

303 How force increases the number of free barbed ends.

304 The force dependent increase in free barbed ends results from a change in the balance between

305 nucleation and capping, driven by a large decrease in capping rate rather than by an increase in

306 nucleation rate. Although the force sensitivity of filament capping has been neglected in most

307 theoretical models of actin network assembly (Mogilner and Oster, 2003; Schaus et al., 2007),

308 we can explain it using Elastic Brownian Ratchet theories developed to describe the effects of

309 force on actin subunit addition during filament elongation (Mogilner and Oster, 1996; Peskin et

310 al., 1993; Theriot, 2000).

312 Since both capping protein and monomeric actin require the same sized gap between the filament

313 barbed end and the boundary it pushes against, the Brownian Ratchet theory predicts that

314 capping and elongation will respond to force in the same way. This means that, when the rate of 
315 filament elongation slows under load, the rate of capping also slows by the same amount.

316 Filaments grow slower but they grow for a proportionally longer time and reach the same

317 average length (Bieling et al., 2016). We directly tested a central prediction of the Brownian

318 Ratchet model by creating a 'bulky' mutant capping protein that requires a larger-sized gap to

319 bind the end of an actin filament. When we applied force to networks that contained the mutant

320 capping protein, the rates of filament elongation and capping diverged sharply. This experiment

321 illustrates the functional importance of the capping protein force response and provides the most

322 direct experimental support for Brownian Ratchet models of force generation to date.

324 The matched force responses of filament elongation and capping suggest that the size of capping

325 protein may be evolutionarily adaptive and subject to positive selection. A mismatch between the

326 sizes of capping protein and actin would cause filaments to lengthen or shorten in response to

327 force, both of which could be deleterious. Filament lengthening would impair force generation

328 because longer filaments more easily bend and/or buckle, while filament shortening would

329 weaken the network by decreasing filament density and network connectivity. In this way, a

330 force-generating Brownian ratchet is controlled by a second, regulatory Brownian ratchet to form

331 a balanced, self-assembly motor. Load-invariance of average filament length also means that

332 this critical network parameter can be tuned independently by polymerases such as formins or

333 Ena/VASP proteins or even WASP-family nucleation promoting factors. 


\section{References:}

Achard V, Martiel J-L, Michelot A, Guérin C, Reymann A-C, Blanchoin L, Boujemaa-Paterski R. 2010. A "primer"-based mechanism underlies branched actin filament network formation and motility. Curr Biol 20:423-428. doi:10.1016/j.cub.2009.12.056

Akin O, Mullins RD. 2008. Capping protein increases the rate of actin-based motility by promoting filament nucleation by the Arp2/3 complex. Cell 133:841-851. doi:10.1016/j.cell.2008.04.011

Bieling P, Hansen SD, Akin O, Li T-D, Hayden CC, Fletcher DA, Mullins RD. 2018. WH2 and proline-rich domains of WASP-family proteins collaborate to accelerate actin filament elongation. EMBO J 37:102-121. doi:10.15252/embj.201797039

Bieling P, Li T-D, Weichsel J, McGorty R, Jreij P, Huang B, Fletcher DA, Mullins RD. 2016. Force Feedback Controls Motor Activity and Mechanical Properties of Self-Assembling Branched Actin Networks. Cell 164:115-127. doi:10.1016/j.cell.2015.11.057

Carlsson AE. 2003. Growth velocities of branched actin networks. Biophys J 84:2907-2918. doi:10.1016/S0006-3495(03)70018-6

Co C, Wong DT, Gierke S, Chang V, Taunton J. 2007. Mechanism of actin network attachment to moving membranes: barbed end capture by N-WASP WH2 domains. Cell 128:901913. doi:10.1016/j.cell.2006.12.049

Dominguez R. 2009. Actin filament nucleation and elongation factors--structure-function relationships. Crit Rev Biochem Mol Biol 44:351-366. doi:10.3109/10409230903277340

Edwards M, Zwolak A, Schafer DA, Sept D, Dominguez R, Cooper JA. 2014. Capping protein regulators fine-tune actin assembly dynamics. Nat Rev Mol Cell Biol 15:677-689. doi:10.1038/nrm3869

Funk J, Merino F, Schaks M, Rottner K, Raunser S, Bieling P. 2021. A structure-derived mechanism reveals how capping protein promotes nucleation in branched actin networks (preprint). Biochemistry. doi:10.1101/2021.03.15.435411

Funk J, Merino F, Venkova L, Heydenreich L, Kierfeld J, Vargas P, Raunser S, Piel M, Bieling P. 2019. Profilin and formin constitute a pacemaker system for robust actin filament growth. Elife 8. doi:10.7554/eLife.50963

Gennerich A, Carter AP, Reck-Peterson SL, Vale RD. 2007. Force-induced bidirectional stepping of cytoplasmic dynein. Cell 131:952-965. doi:10.1016/j.cell.2007.10.016

Helgeson LA, Nolen BJ. 2013. Mechanism of synergistic activation of Arp2/3 complex by cortactin and N-WASP. Elife 2:e00884. doi:10.7554/eLife.00884

Husson C, Cantrelle F-X, Roblin P, Didry D, Le KHD, Perez J, Guittet E, Van Heijenoort C, Renault L, Carlier M-F. 2010. Multifunctionality of the beta-thymosin/WH2 module: Gactin sequestration, actin filament growth, nucleation, and severing. Ann N Y Acad Sci 1194:44-52. doi:10.1111/j.1749-6632.2010.05473.x

Kim T, Cooper JA, Sept D. 2010. The interaction of capping protein with the barbed end of the actin filament. J Mol Biol 404:794-802. doi:10.1016/j.jmb.2010.10.017

Laakso JM, Lewis JH, Shuman H, Ostap EM. 2008. Myosin I can act as a molecular force sensor. Science 321:133-136. doi:10.1126/science.1159419

Loisel TP, Boujemaa R, Pantaloni D, Carlier MF. 1999. Reconstitution of actin-based motility of Listeria and Shigella using pure proteins. Nature 401:613-616. doi:10.1038/44183

Marcy Y, Prost J, Carlier M-F, Sykes C. 2004. Forces generated during actin-based propulsion: a direct measurement by micromanipulation. Proc Natl Acad Sci U S A 101:5992-5997. doi:10.1073/pnas.0307704101 
382

383

384

385

386

387

388

389

390

391

392

393

394

395

396

397

398

399

400

401

402

403

404

405

406

407

408

409

410

411

412

413

414

415

416

417

418

419

420

421

422

423

424

425

426

427

Mogilner A, Oster G. 2003. Force generation by actin polymerization II: the elastic ratchet and tethered filaments. Biophys J 84:1591-1605. doi:10.1016/S0006-3495(03)74969-8

Mogilner A, Oster G. 1996. Cell motility driven by actin polymerization. Biophys J 71:30303045. doi:10.1016/S0006-3495(96)79496-1

Mueller J, Szep G, Nemethova M, de Vries I, Lieber AD, Winkler C, Kruse K, Small JV, Schmeiser C, Keren K, Hauschild R, Sixt M. 2017. Load Adaptation of Lamellipodial Actin Networks. Cell 171:188-200.e16. doi:10.1016/j.cell.2017.07.051

Mullins RD, Bieling P, Fletcher DA. 2018. From solution to surface to filament: actin flux into branched networks. Biophys Rev 10:1537-1551. doi:10.1007/s12551-018-0469-5

Mullins RD, Heuser JA, Pollard TD. 1998. The interaction of Arp2/3 complex with actin: nucleation, high affinity pointed end capping, and formation of branching networks of filaments. Proc Natl Acad Sci U S A 95:6181-6186. doi:10.1073/pnas.95.11.6181

Narita A, Takeda S, Yamashita A, Maéda Y. 2006. Structural basis of actin filament capping at the barbed-end: a cryo-electron microscopy study. EMBO J 25:5626-5633. doi:10.1038/sj.emboj.7601395

Pandit NG, Cao W, Bibeau J, Johnson-Chavarria EM, Taylor EW, Pollard TD, De La Cruz EM. 2020. Force and phosphate release from Arp2/3 complex promote dissociation of actin filament branches. Proc Natl Acad Sci U S A 117:13519-13528. doi:10.1073/pnas.1911183117

Parekh SH, Chaudhuri O, Theriot JA, Fletcher DA. 2005. Loading history determines the velocity of actin-network growth. Nat Cell Biol 7:1219-1223. doi:10.1038/ncb1336

Peskin CS, Odell GM, Oster GF. 1993. Cellular motions and thermal fluctuations: the Brownian ratchet. Biophys J 65:316-324. doi:10.1016/S0006-3495(93)81035-X

Rohatgi R, Ma L, Miki H, Lopez M, Kirchhausen T, Takenawa T, Kirschner MW. 1999. The interaction between N-WASP and the Arp2/3 complex links Cdc42-dependent signals to actin assembly. Cell 97:221-231. doi:10.1016/s0092-8674(00)80732-1

Schafer DA, Jennings PB, Cooper JA. 1996. Dynamics of capping protein and actin assembly in vitro: uncapping barbed ends by polyphosphoinositides. J Cell Biol 135:169-179. doi:10.1083/jcb.135.1.169

Schaus TE, Taylor EW, Borisy GG. 2007. Self-organization of actin filament orientation in the dendritic-nucleation/array-treadmilling model. Proc Natl Acad Sci U S A 104:7086-7091. doi:10.1073/pnas.0701943104

Smith BA, Padrick SB, Doolittle LK, Daugherty-Clarke K, Corrêa IR, Xu M-Q, Goode BL, Rosen MK, Gelles J. 2013. Three-color single molecule imaging shows WASP detachment from Arp2/3 complex triggers actin filament branch formation. Elife 2:e01008. doi:10.7554/eLife.01008

Theriot JA. 2000. The polymerization motor. Traffic 1:19-28.

Weichsel J, Schwarz US. 2010. Two competing orientation patterns explain experimentally observed anomalies in growing actin networks. Proc Natl Acad Sci U S A 107:63046309. doi:10.1073/pnas.0913730107

Yildiz A, Tomishige M, Gennerich A, Vale RD. 2008. Intramolecular strain coordinates kinesin stepping behavior along microtubules. Cell 134:1030-1041. doi:10.1016/j.cell.2008.07.018

Zalevsky J, Lempert L, Kranitz H, Mullins RD. 2001. Different WASP family proteins stimulate different Arp2/3 complex-dependent actin-nucleating activities. Curr Biol 11:1903-1913. doi:10.1016/s0960-9822(01)00603-0 

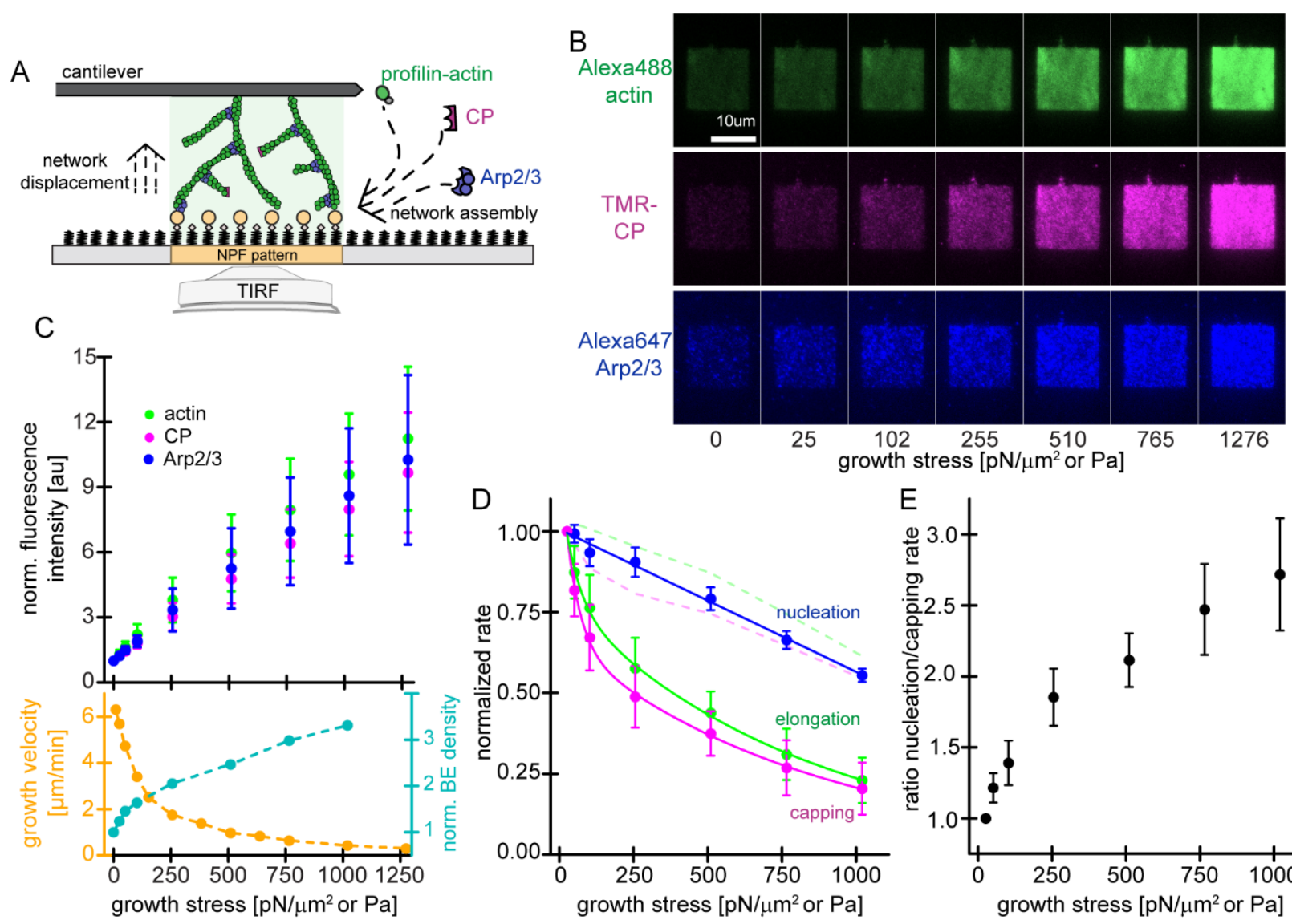

Figure 1. Effect of mechanical load on Arp2/3-dependent nucleation. A) Schematic

illustration of actin networks generated by profilin-actin, the Arp2/3 complex and capping protein from surfaces coated with NPF (WAVE1 $\Delta \mathrm{N})$. Conditions are $5 \mu \mathrm{M}$ actin (1\% Alexa 488labeled), $5 \mu \mathrm{M}$ profilin, 100nM Arp2/3 (5\% Alexa647-labeled), 100nM CP (15\% TMR-labeled) if not indicated otherwise. B) TIRFM images of Alexa488-actin (top), TMR-CP (middle) and Alexa647-Arp2/3 (bottom) incorporation into dendritic actin networks at indicated growth stress. C) Top: Quantification of florescent intensities for indicated network components as a function of applied load. Bottom: Corresponding growth velocities and free barbed end densities as measured in (Bieling et al., 2016). Error bars are SD. D) Average rates of filament elongation,

440 capping and nucleation (calculated by the product of the bulk fluorescence intensities and the 441 network growth velocity, normalized to the flux at $25 \mathrm{pN} / \mu \mathrm{m} 2$ ) as a function of external load.

442 Capping and elongation rates were obtained by normalizing their network incorporation rates 443 (dashed lines) by the relative density of free barbed ends as shown in C). Error bars are SEM. E)

444 Ratio of nucleation and capping rates as a function of external load. Error bars are SEM. 

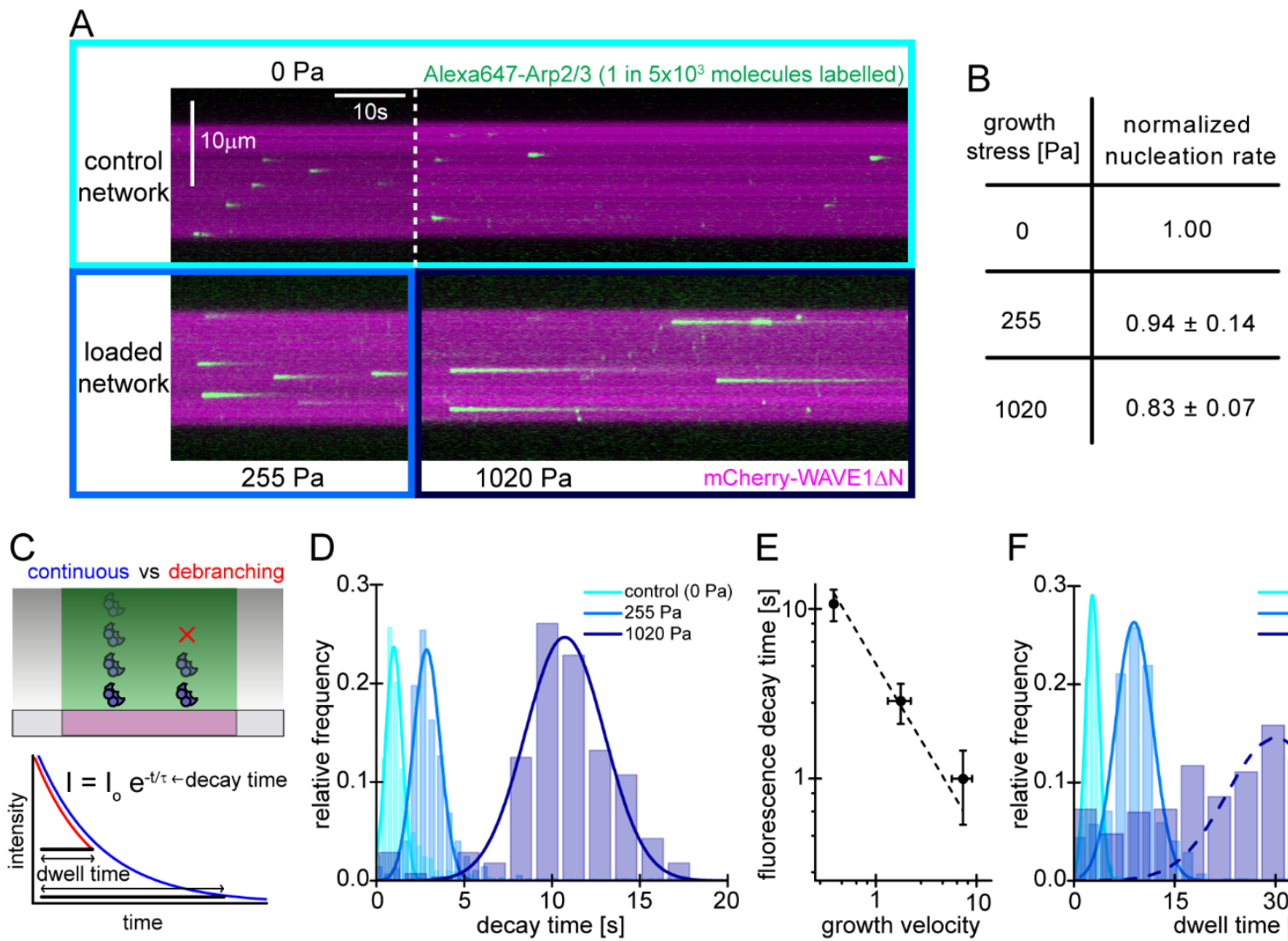

$\mathrm{E}$

G

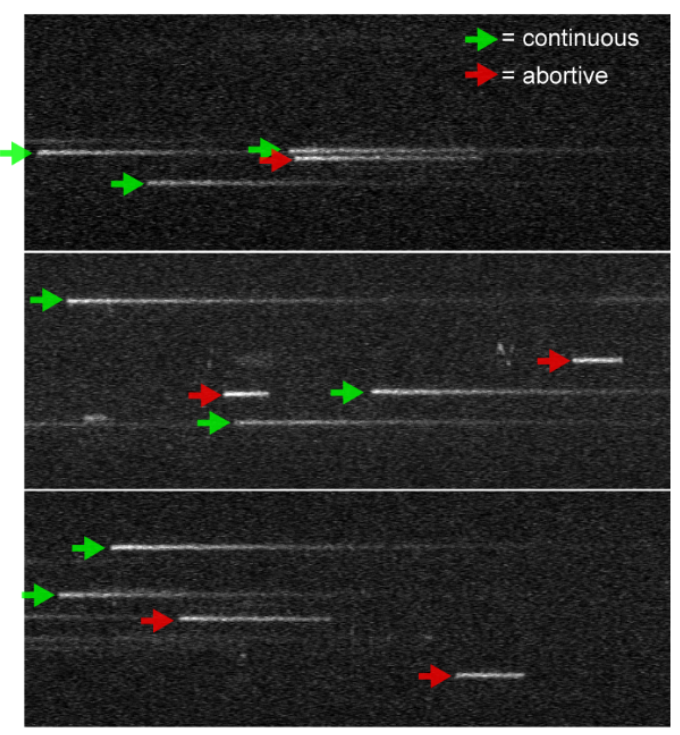

$\mathrm{H}$
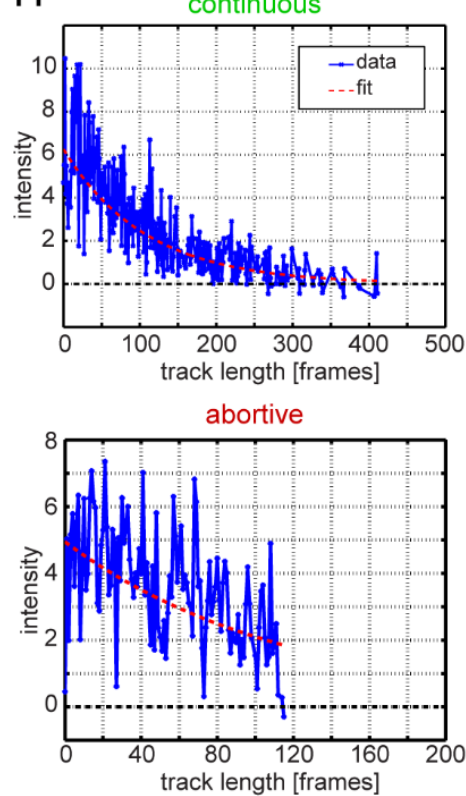

$\mathrm{F}$
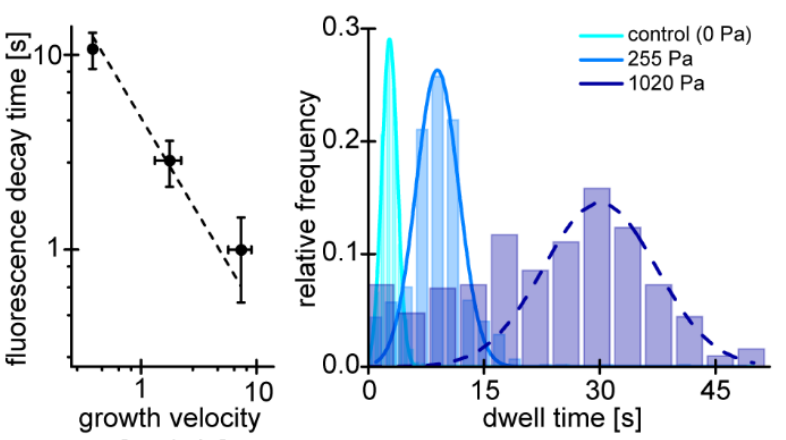

$[\mu \mathrm{m} / \mathrm{min}]$ magenta) by spike-in of a small fraction of Alexa647-Arp2/3 (green, c=20 pM) into the overall 
450 network (upper panel). B) Mean nucleation rate by single molecule imaging normalized to the

451 nucleation rate in an adjacent unloaded control network at indicated growth stress. Error bars are

452 SEM. C) Scheme of single molecule Arp2/3 nucleation by TIRFM for either continuous (blue)

453 or debranching (red) scenarios. Individual intensity trajectories are characterized by either their

454 transit time through the evanescent field or their actual dwell time. The latter is sensitive to

455 debranching. D) Normalized frequency of fluorescence transit times of single Alexa 647-Arp2/3

456 molecules in networks assembled at indicated stress. Continuous lines are Gaussian fits to the

457 data. E) Double-logarithmic plot of the mean fluorescence transit time (+/- SD) as a function of

458 network growth velocity. The dashed line show perfect reciprocal correlation (slope $=-1)$. F)

459 Normalized frequency of fluorescence dwell times of single Alexa 647-Arp2/3 molecules in

460 networks assembled at indicated stress. Continuous lines are Gaussian fits to the data. Dashed

461 line is a Gaussian fit to a partial (normally distributed) fraction of the $1020 \mathrm{pN} / \mu \mathrm{m} 2$ data set. G)

462 Examples of kymographs from TIRF microscopy of individual Arp2/3 molecules in networks

463 under high load $(1020 \mathrm{pN} / \mathrm{m} 2)$. Individual complexes are either continuously moving towards the

464 rear of the evanescent field (continuous, green arrows) or dissociating prematurely (abortive, red

465 arrows). H) Representative time courses of fluorescence intensity for individual Arp2/3

466 complexes as a function of number of imaging frames categorized as either continuous (top

467 panel) or abortive (bottom panel). I) Relative frequency of dwell times for Arp2/3 molecules in

468 dendritic networks at high load $(1020 \mathrm{pN} / \mathrm{m} 2$, blue) compared to the bleaching and loss of

469 tracking control (red, see Supplementary Figure 1). Note that the frequency of early loss events

470 is exceeding the combined bleaching and tracking loss frequency. 
A
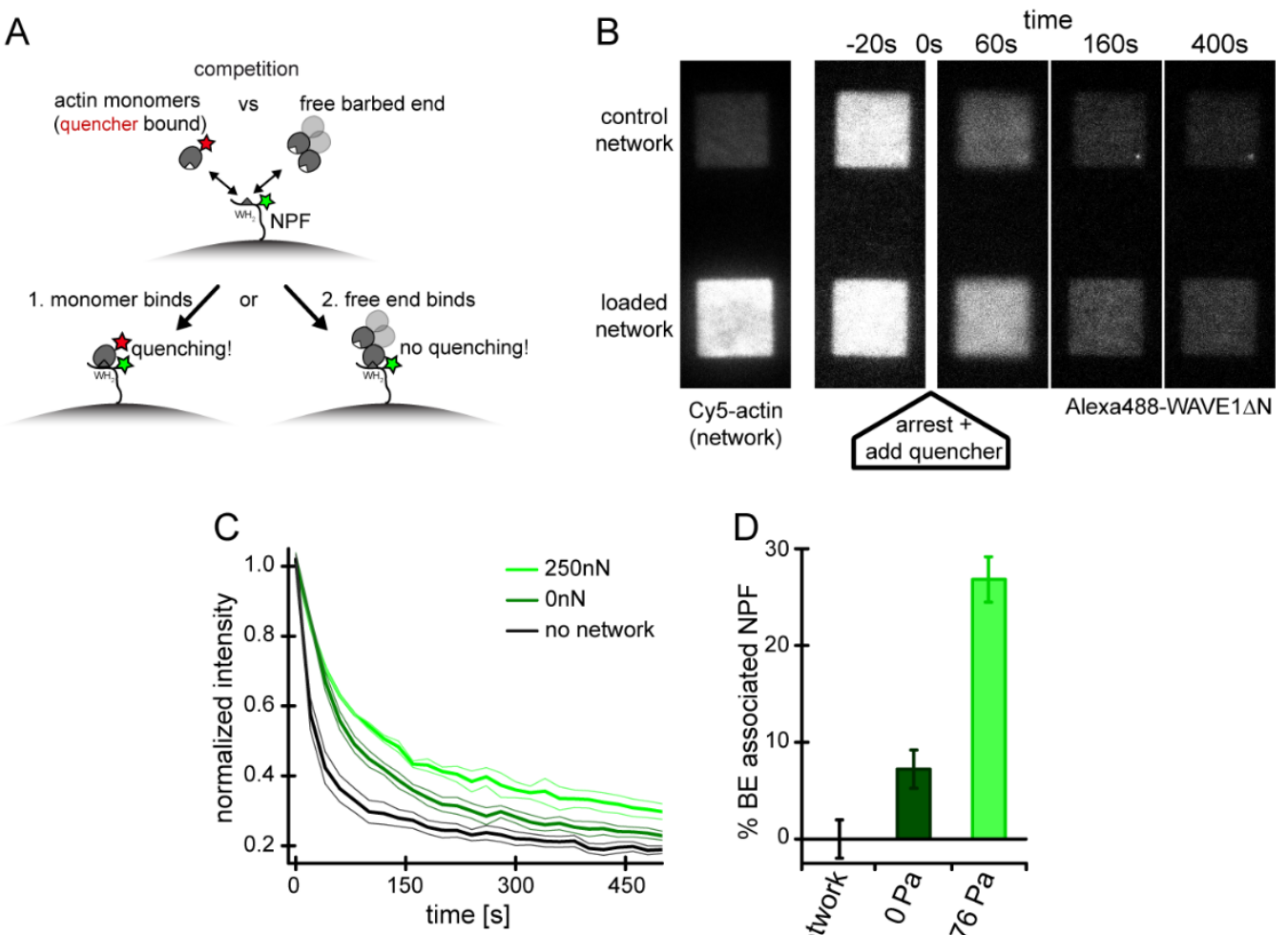

473

Figure 3. Free barbed ends attach to and sequester the WH2 domain of the NPF in a loaddependent manner. A) Scheme of the FRET setup. Surface-bound, donor- (Alexa488-) labeled NPF molecules can interact with either quencher- (Atto540Q-) labeled actin monomers resulting in decrease of donor fluorescence or unlabeled terminal protomers of uncapped barbed ends resulting in no change in fluorescence. The terminal protomers are unlabeled since quencherlabeled monomers are introduced only upon network arrest. B) Time lapse TIRF microscopy images of Alexa 647-Actin (left image) or Alexa 488-WAVEAN (FRET donor, other images) at indicated times after addition of $200 \mu \mathrm{l}$ fixation and quenching mix $(\mathrm{t}=0,30 \mu \mathrm{M}$ LatB, 30 $\mu \mathrm{M}$ phalloidin, $5 \mu \mathrm{M}$ Atto540Q-actin, $7 \mu \mathrm{M}$ profilin, $37.5 \mu \mathrm{M}$ myotrophin/V1 (CP inhibitor)) to 100 $\mu \mathrm{l}$ network assembly mix.

C) Time-courses of the Alexa 488-WAVE $\Delta \mathrm{N}$ signal following the addition of quencher-labelled monomers at $\mathrm{t}=0$ as shown in B) Error indicators are SD. D) Mean fraction of barbed end-associated NPF molecules in either in the absence of an actin network (black) or in the presence of a non-loaded (dark green) or $200 \mathrm{nN}$ loaded (light green) network (See Supplemental Methods). Error bars are SEM. 

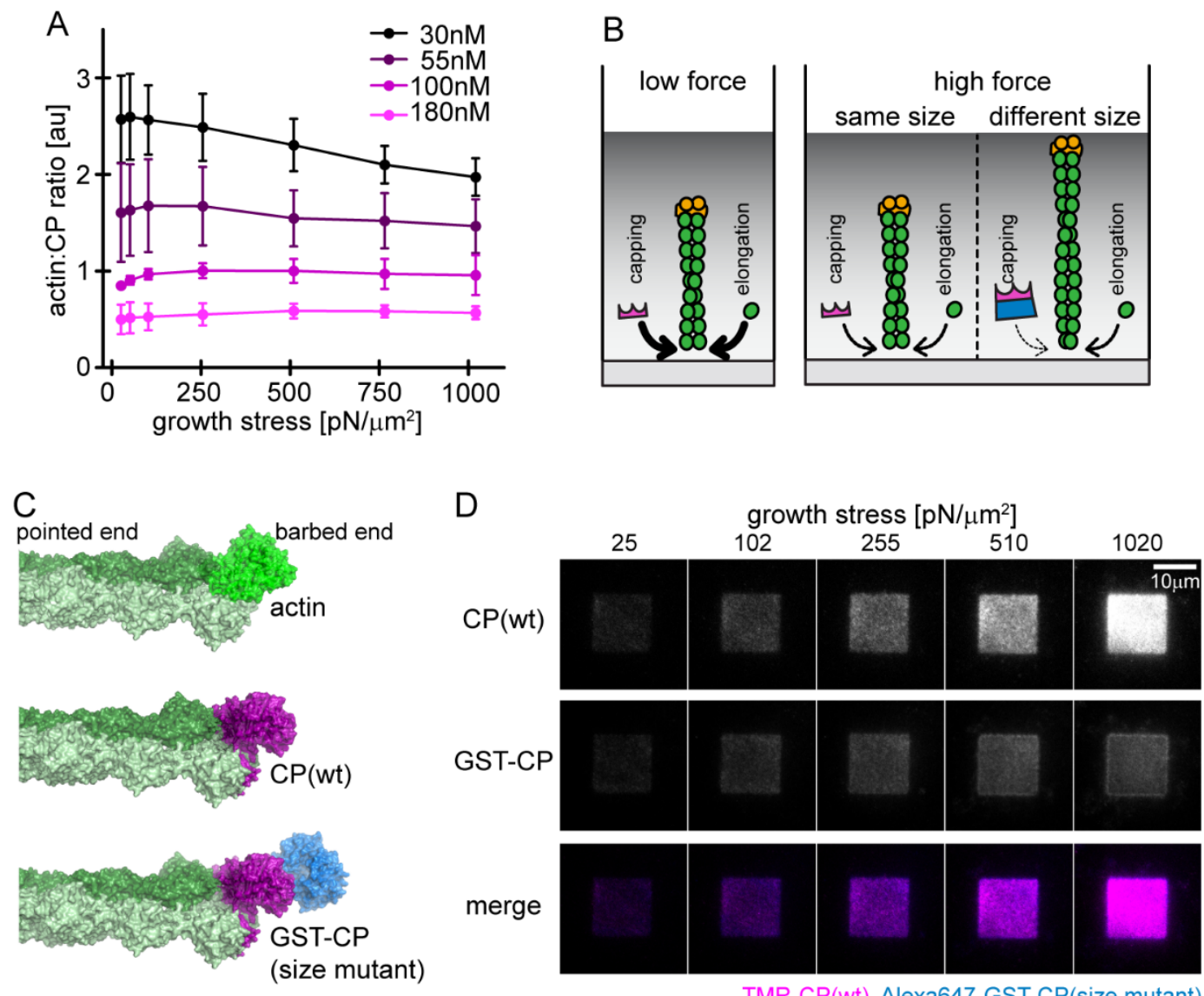

GST-CP

$\mathrm{E}$
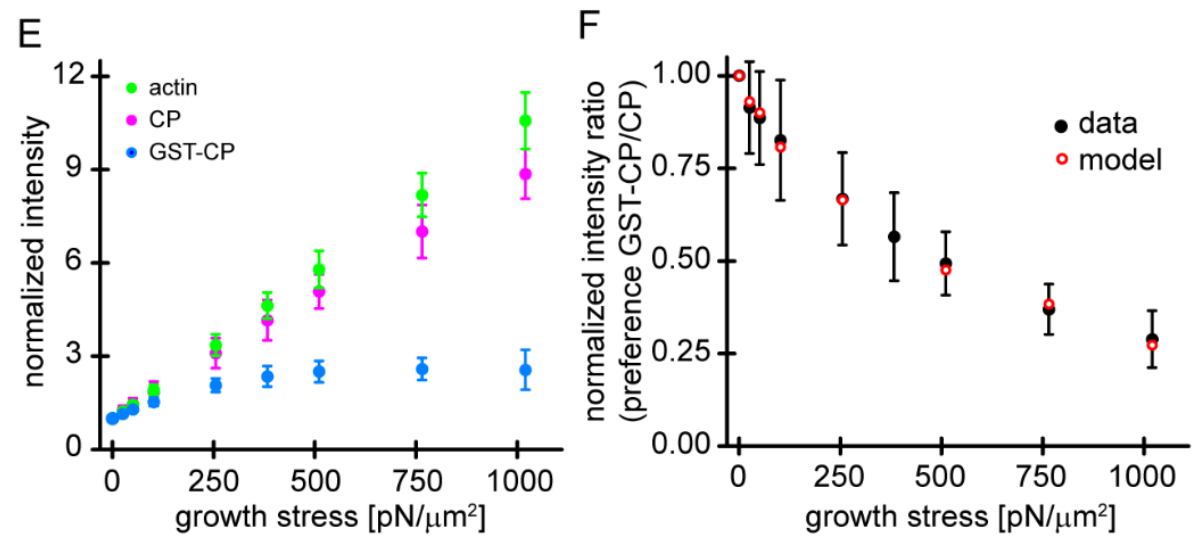

Figure 4. Load dependence of capping and a direct test of the elastic Brownian theory of force generation by actin networks. A) Ratios of capping protein to actin fluorescence for networks grown at different CP concentrations as indicated as a function of load (see Supplemental Figure 3). Error bars are SD. B) Illustration of the consequences of load dependence of capping and polymerization. Low load allows for high capping and polymerization rates (left panel). A similar load dependence of these two processes maintains filament length at high load (middle panel), whereas a difference in load dependence leads to 
502 changes in filament length (right panel). C) Structural models of a filament barbed end (light and

503 dark green) bound by either an additional actin monomer (top panel, bright green), a wt CP

504 heterodimer (middle panel, magenta) or an engineered GST and CP dimer fusion ("bulky

505 variant", bottom panel, magenta=CP, blue=GST). D) TIRFM images of dendritic actin networks

506 (top panel=TMR-CP (wt), middle panel=Alexa647-GST-CP (bulky variant) and bottom

507 panel=color merge) at indicated stress. Networks were assembled at standard conditions, except

508 that CP (wt) concentration was 90nM (of which 10nM were TMR-CP) and Alexa647-GST-CP

509 concentration was 10nM. E) Mean Alexa 488-actin, TMR-CP(wt) or Alexa 647-GST-CP (bulky

510 variant) intensity normalized to the intensity of an adjacent unloaded network as a function of

511 load. Error bars are SD. F) Measured mean fluorescence intensity ratios of CP(wt)/GST-

$512 \mathrm{CP}$ (bulky variant) normalized to the intensity ratio of an adjacent unloaded network as a function

513 of load. Error bars are SD. Red open circles are derived from the Brownian Ratchet Model (see

514 Appendix). 